\title{
Aberration-corrected HRTEM of defects in strained lanthanum cuprate thin films grown on strontium titanate
}

\section{Houben}

Ernst Ruska-Centre for Microscopy and Spectroscopy with Electrons (ER-C) and Institute of Solid State Research, Research Centre Jülich, 52425 Jülich, Germany

The structure of lattice defects in $\mathrm{La}_{2} \mathrm{CuO}_{4}(\mathrm{La} 214)$ thin films grown under tensile strain on $\mathrm{SrTiO}_{3}$ (STO) is investigated by the combination of state-of-the-art medium voltage aberration-corrected transmission electron microscopy at $200 \mathrm{kV}$ [1,2] together with numerical exit-plane wavefunction reconstruction from focal series [3,4]. The interfacial reconstruction, the coordination in planar shear defects evolving from surface steps and the core of misfit dislocations are atomically resolved and analysed. Directly interpretable contrast features coming along with the widely minimised image delocalisation associated with aberration correction enable for the identification and proper quantification of the structural reconstructions. The quantitative evaluation, e.g. in terms of identification of atom types or vacant sites and the precise measurement of atom column displacements, demonstrates the prospect of success in targeting at the evaluation of the electronic reconstruction at such defects on the atomic scale, especially when combining such analyses with complementary analytical work and theoretical calculations.

A first example is the structural reconstruction at the interface between La214 and STO exposed in Fig. 1 by a single experimental image taken at negative spherical aberration imaging conditions (NCSI) [5] and the phase of the exit-plane wavefunction retrieved from a focal series. Both images unveil the atomic coordination at the interface with bright dots corresponding to atom columns of cations and oxygen, exemplarily illustrated by the excellent agreement between structure model, simulation and experimental phase image in Figs. $1 \mathrm{~b}$ and c. The quantitative mapping and evaluation of peak data in the phase image reveals the formation of a perovskite-like layer of lanthanum copper oxide (Fig. 2), analogous to the thermodynamically instable $\mathrm{LaCuO}_{3-\delta}$ phase, and a distortion in the octahedral coordination of copper at the interface to the substrat. Fig. 3 shows as a second example the structural reconstruction at planar shear defects originating from steps on the substrate surface of the size o a perovskite block. A detailled model of the structural coordination given in Figs. $3 b$ and $c$ exhibits peculiar features in of the projected structure which are found in one-to-one correspondence in the NCSI image in Fig. 3a. In particular, the planar shear defects embody extra sites for cations and oxygen in a three-dimensional periodic arrangement which are partially filled and provide paths for vacancy hopping transport. A further example investigates the core structure of a $a[010]$ type misfit dislocation. Here, the quantitative evaluation of phase peaks in exit-plane wavefunctions is used to map the displacement field in the cation sublattice of the highly strained dislocation centre with an accuracy of $\sim 10 \mathrm{pm}$ and to identify the cation type on a specific atom site where statistical significance can be achieved.

\section{References}

[1] M. Haider, H. Rose, S. Uhlemann, E. Schwan, B. Kabius and K. Urban, Nature 392 (1998) 768. [2] M. Lentzen, B. Jahnen, C.L. Jia, K. Tillmann and K. Urban, Ultramicroscopy 92 (2002) 233.

[3] W. Coene, A. Thust, M. Op de Beeck and D. Van Dyck, Ultramicroscopy 64 (1996) 109. 
[4] A. Thust, W. Coene, M. Op de Beeck And D. Van Dyck, Ultramicroscopy 64 (1996) 211.

[5] C.L. Jia, M. Lentzen, K. Urban, Science 299 (2003) 870.
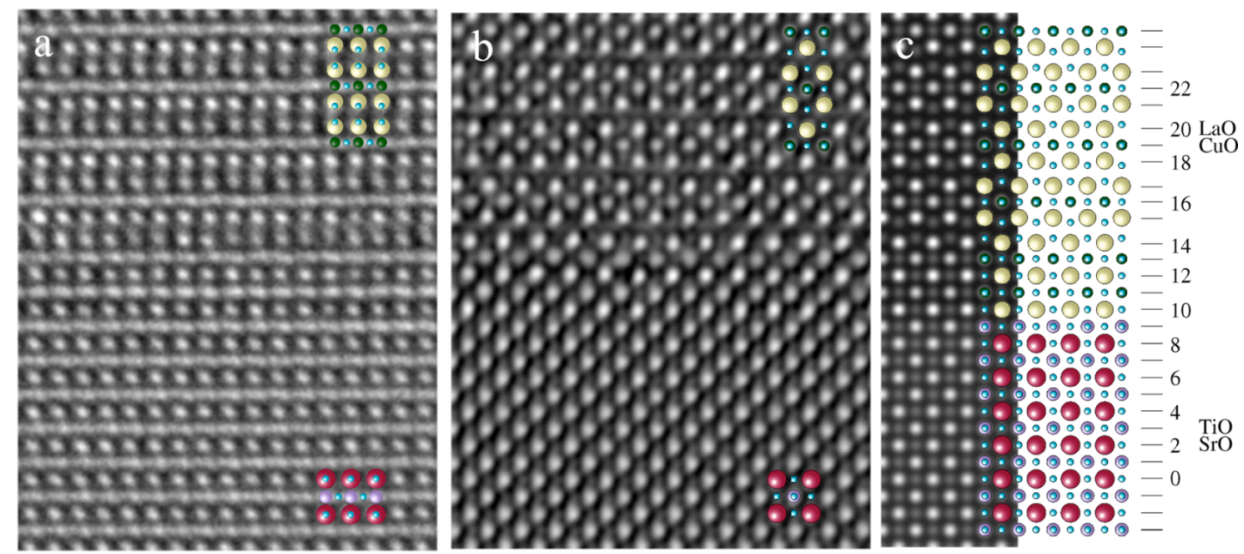

Fig. 1: Atomic structure of the La214/STO interface. (a) NCSI image taken along the [110] viewing direction. (b) Phase of the exit-plane wavefunction reconstructed from a focal series taken in the [100] zone axis. (c) Structure model superimposing the phase of the simulated exit-plane wavefunction along the [100] viewing direction.

Fig. 2: Peak phase on cation atom column sites in successive layers across the La214/STO interface. A single data point represents the average peak phase for 15 equivalent sites in a monolayer. A single peak value was evaluated by gaussian regression to a phase maximum in Fig. 1(b). The error estimates correspond to two times the standard deviation of the 15 point data set.
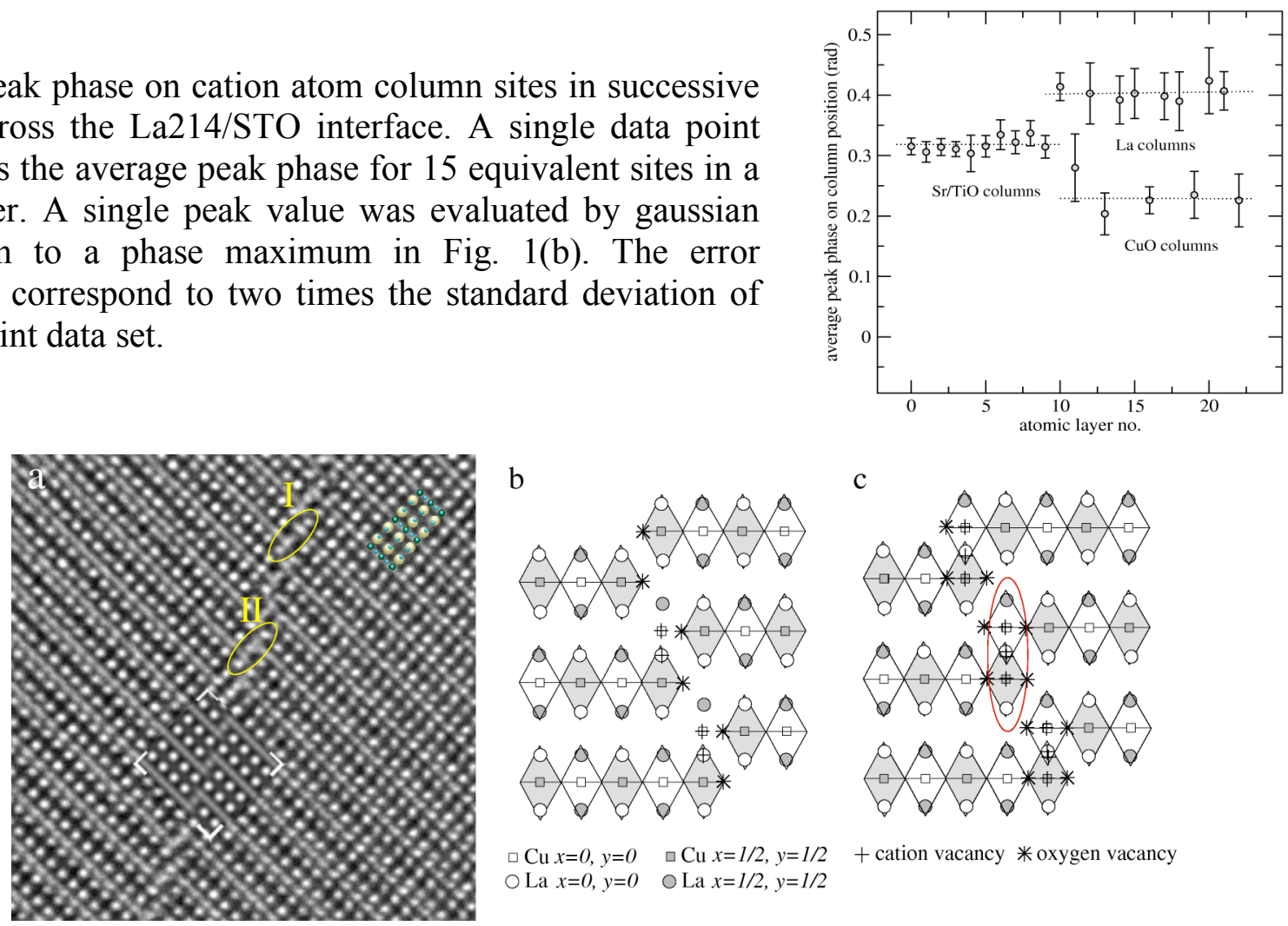

Fig. 3: (a) Exemplary NCSI image of a shear defect in La214 taken along the [110] viewing direction. The framed part shows a simulated NCSI image for the structure model outlined in (b) and (c). (b) Detailed drawing of the layer breaking across a shear defect along the [110] viewing direction for a layer with a thickness of one unit cell. (c) Projected structure of the defect in two successive unit cell layers, which are shifted by the size of a $\mathrm{CuO}_{6}$ octahedron. The repeating group of five cation columns found e.g. in regions I and II in (a) is highlighted. 